\title{
Role of the illumination spatial-frequency spectrum for ptychography
}

\author{
Manuel Guizar-Sicairos, ${ }^{*}$ Mirko Holler, Ana Diaz, Joan Vila-Comamala, ${ }^{\dagger}$ Oliver Bunk, and Andreas Menzel \\ Paul Scherrer Institut, 5232 Villigen PSI, Switzerland \\ (Received 30 July 2012; published 24 September 2012)
}

\begin{abstract}
We demonstrate how the spatial-frequency spectrum of the x-ray illumination affects the reconstruction signal-to-noise ratio and resolution of ptychographic imaging. The spatial-frequency spectrum of a focused $\mathrm{x}$-ray probe is enhanced by partially clipping the beam with an aperture near its focus. This approach presents a simple way of enhancing the illumination spectrum without demanding extra efforts in optics fabrication, and we experimentally demonstrate that it provides an improvement in image quality and resolution.
\end{abstract}

DOI: 10.1103/PhysRevB.86.100103

PACS number(s): 42.30.Rx, 68.37.Yz, 41.50.+h, 42.25.Fx

Ptychography, a coherent diffractive imaging (CDI) technique, is rapidly evolving into a microscopy method useful for quantitative imaging in two ${ }^{1,2}$ and three dimensions. ${ }^{3,4}$ The technique combines scanning microscopy and iterative reconstruction algorithms to obtain a resolution much finer than the size of the illuminating probe and the scanning step. The success of the technique stems from the diversity of measurements ${ }^{5,6}$ that improves the image reconstruction convergence and success rate. Additionally, the need for an isolated sample, characteristic of plane-wave $\mathrm{CDI},{ }^{7}$ is removed, and a customized field of view can be imaged, thus simplifying sample preparation and making the technique applicable to a wide range of scientific questions.

A key feature of ptychography is its relaxed requirements on the illuminating probe. The probe should be transversely and longitudinally coherent, and its transverse size should be small enough so that the far-field intensity patterns can be adequately sampled by the detector. Aside from this, the amplitude and phase of the probe are quite arbitrary. In fact, this property allows application of ptychography as a beam characterization ${ }^{8,9}$ and wave front aberration sensing technique. ${ }^{10-12}$ For imaging, this presents an opportunity to explore what are the characteristics of the illumination that are beneficial for the image reconstruction. The measured intensities at a given scattering angle depend on the scattering power of the object of interest and on the spatial-frequency spectrum of the illuminating beam. Since the former is fixed by the specimen under study it is the latter that amounts to a useful degree of freedom.

In its original framework, a closed-form solution to ptychography can be obtained through Wigner deconvolution if diffraction patterns are taken with fine sample steps, equal to or better than the sought resolution. ${ }^{13}$ While this is experimentally impractical, this linear and closed-form procedure proves useful to understand the mechanism of information encoding by ptychography. After a four-dimensional Fourier transform, along the detector pixel coordinates (q space) and along the object positions, the data are expressed as the product $\chi_{o}(\mathbf{r}, \mathbf{q}) \chi_{p}(\mathbf{r},-\mathbf{q}),{ }^{13}$ where

$$
\chi_{o}(\mathbf{r}, \mathbf{q})=\int o(\mathbf{c}) o(\mathbf{c}+\mathbf{r}) \exp (i 2 \pi \mathbf{c} \cdot \mathbf{q}) \mathrm{d} \mathbf{c}
$$

is the ambiguity function of the object complex-valued transmissivity, $o(\mathbf{r}), \chi_{p}(\mathbf{r}, \mathbf{q})$ is the probe ambiguity function, and $\mathbf{r}$ and $\mathbf{q}$ are the two-dimensional Cartesian coordinates in real

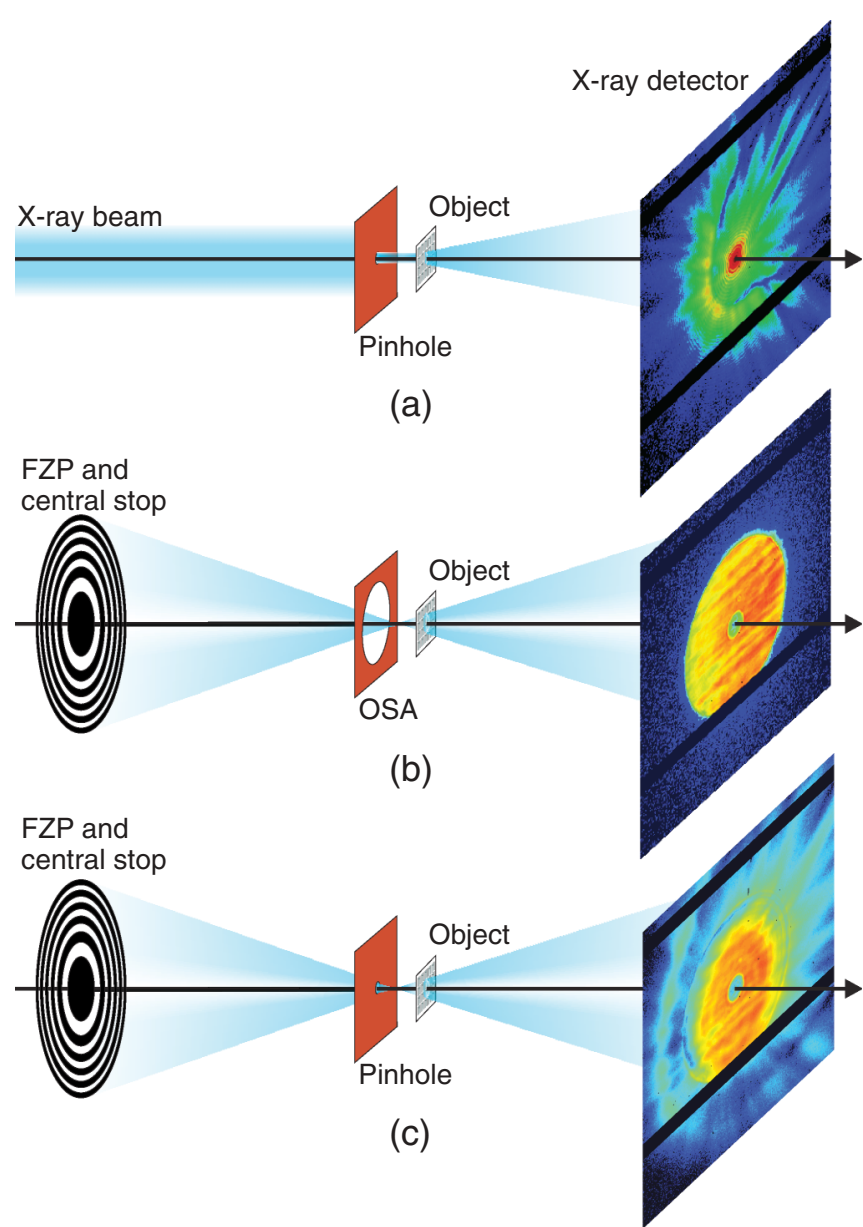

FIG. 1. (Color online) Experimental setup allowing three illuminations: (a) pinhole alone, (b) FZP focused beam, and (c) FZP focus with a pinhole perturbation.

and reciprocal space, respectively. The object transmissivity can be reconstructed from $\chi_{o}(\mathbf{r}, \mathbf{q})$ which is estimated after a point-by-point division, ${ }^{13}$ or in the presence of noise and low amplitudes of $\chi_{p}(\mathbf{r}, \mathbf{q})$ by application of a Wiener filter. ${ }^{14}$ The object can then be recovered to a resolution beyond a limited probe spectrum through a "stepping-out" procedure. ${ }^{13}$ Because of the product of the ambiguity functions, the signalto-noise ratio (SNR) of the estimated $\chi_{o}(\mathbf{r}, \mathbf{q})$, and thus of the reconstructed object, depends on the amplitude of the probe ambiguity function. While a limited probe spectrum 

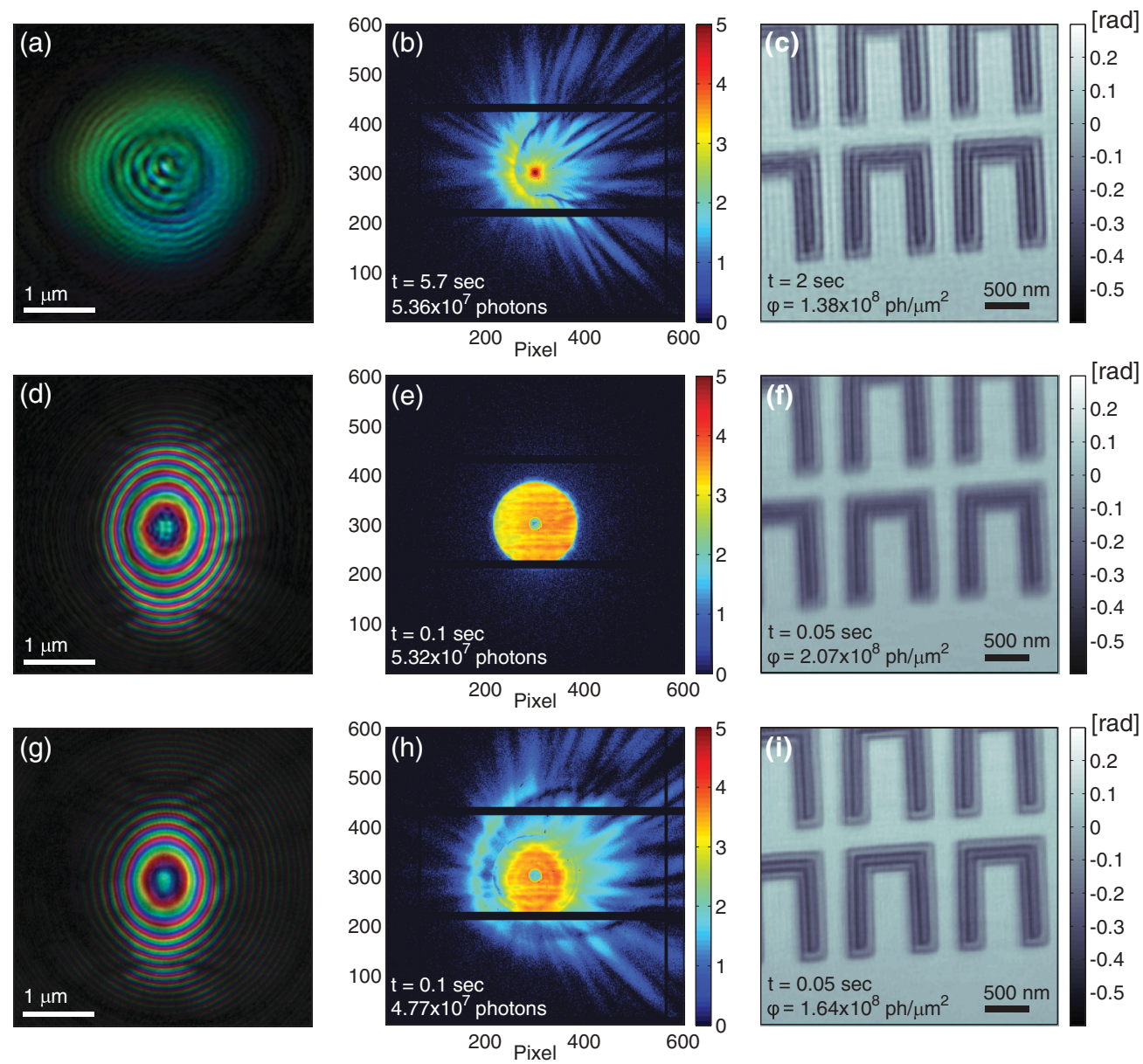

FIG. 2. (Color online) (a) Complex-valued representation of the reconstructed pinhole illumination at the plane of the object where brightness and hue represent amplitude and phase, respectively. (b) Pinhole diffraction pattern without an object on a base-10 logarithmic scale with the exposure time and total photon count indicated in the figure. (c) $3 \times 3 \mu \mathrm{m}^{2}$ inset of the phase of the reconstruction using the pinhole illumination. Exposure time per point and total photon density for this measurement are indicated on the figure. (d)-(f) Same as above but for the FZP illumination. (g)-(h) Same as above but for the FZP illumination with a pinhole partially clipping the beam. Total exposure time, neglecting overhead, for a $5 \times 5 \mu \mathrm{m}^{2}$ was (c) 332 seconds, (f) and (i) 8.3 seconds.

does not pose a fundamental limitation in resolution but rather a disadvantage in SNR, for a faithful recovery of the object's high-resolution details it helps to have a probe ambiguity function with a wide spread in reciprocal space.

To experimentally study the role of the spatial-frequency spectrum of the probe for ptychography we imaged a test structure lithographically made from hydrogen silsesquioxane resist (HSQ) and coated with a $17 \mathrm{~nm}$ layer of iridium. ${ }^{15}$ $\mathrm{X}$-ray experiments were performed at the cSAXS beamline (X12SA), Swiss Light Source, and the Fresnel zone plate (FZP) and test structure were fabricated at the Laboratory for Micro- and Nanotechnology, both at the Paul Scherrer Institut, Switzerland. An x-ray energy of $6.2 \mathrm{keV}(\lambda=0.2 \mathrm{~nm})$ was used for three different illuminations, as shown in Fig. 1, with similar transverse size of about $2.5 \mu \mathrm{m}$ at the object plane but distinctly different spatial-frequency spectra. For the measurements the object was scanned to 83 different transverse positions along concentric circles with a radial spacing of $500 \mathrm{~nm}$ and covering a field of view of $5 \times 5 \mu \mathrm{m}^{2}$. At each point a diffraction pattern was measured by a photoncounting detector with $172 \mu \mathrm{m}$ pixel size located $7.2 \mathrm{~m}$ downstream of the object. ${ }^{16}$ The measurement was repeated at two detector positions in order to measure the data lost due to detector gaps, which are evident in Figs. 2(b), 2(e), and 2(h). The reconstruction was performed using a difference map algorithm ${ }^{2,17}$ using $600 \times 600$ detector pixels, which corresponds to a reconstruction pixel size of $14 \mathrm{~nm}$, and treating the diffraction patterns from the two detector positions as suitably masked independent measurements. ${ }^{12}$

The first probe, shown in Fig. 2(a), was defined by a $2.5 \mu \mathrm{m}$ pinhole placed $1.5 \mathrm{~mm}$ upstream of the object. The high-contrast and relatively sharp edges of the pinhole provide a spatial spectrum that follows a power law and is suitable for imaging over a wide range of flux, but also requires a high dynamic range detector due to the concentration of intensity in the forward direction, and makes it difficult to collect the coherent flux available at the experimental station if not combined with a prefocusing scheme. Figure 2(b) shows a diffraction pattern of the pinhole. The reconstruction using a 2 second exposure time per diffraction pattern, shown in Fig. 2(c), shows high-resolution details but also suffers from some artifacts that can be seen on the background, which is 
expected to be uniform. These artifacts may be related to stage drifts or small changes of the probe during the scan.

The second probe, shown in Fig. 2(d), was defined by removing the pinhole and introducing a gold Fresnel zone plate $^{18}$ of $200 \mu \mathrm{m}$ diameter and focal length of $50 \mathrm{~mm}$. The zone plate had a $50 \mathrm{~nm}$ outermost zone width and a $500 \mathrm{~nm}$ zone height and its focus was positioned $0.5 \mathrm{~mm}$ upstream of the object in order to create a beam of transverse size comparable to the pinhole. In front of the FZP a $30 \mu \mathrm{m}$ central stop was introduced as well as a $20 \mu \mathrm{m}$ order-sorting aperture (OSA) which was mounted $1.5 \mathrm{~mm}$ upstream of the object, as shown in Fig. 1(b). A vertical slit located $22 \mathrm{~m}$ upstream of the FZP was set to a horizontal width of $12.5 \mu \mathrm{m}$ to ensure that the FZP was coherently illuminated. Despite the losses due to the FZP diffraction efficiency the flux increased by a factor close to 60 compared to the pinhole. To have a comparable photon density during the measurements we reduced the exposure time to 0.05 seconds per diffraction pattern and consequently made the measurement using the FZP probe faster. An additional advantage is that instabilities on the upstream beamline optics have a reduced effect over the $200 \mu \mathrm{m}$ aperture of the FZP compared to the $2.5 \mu \mathrm{m}$ diameter of the pinhole. Both effects result in a significant improvement on background artifacts in the reconstruction in Fig. 2(f). However, the maximum angular spread of the FZP beam is limited by the outer zone width and drops quite suddenly as shown in Fig. 2(e). Hence, Fig. 2(f), due to the narrower probe spectrum, shows a reduced sharpness of the image, despite the increased photon density compared to Fig. 2(c).

The introduction of phase curvature in the illumination is used in Fresnel CDI to improve robustness and convergence of the iterative algorithm, ${ }^{19}$ and similar benefits apply to ptychography through the diversity in measurements introduced by overlapped probe positions. ${ }^{5}$ Figures $2(\mathrm{c})$ and 2(f), in agreement with numerical simulations, suggest that the combination of both yields on its own no additional benefits.

In order to increase the spectral width of the FZP illumination, a third probe was created by replacing the OSA with the $2.5 \mu \mathrm{m}$ pinhole located at $1.5 \mathrm{~mm}$ upstream of the object and $1 \mathrm{~mm}$ upstream of the focus, such that the focused beam was partially clipped. This third configuration is depicted in Fig. 1(c). The pinhole reduced the photon flux by about $10 \%$, and although the probe on Fig. 2(g) remains similar in appearance to Fig. 2(d), the pinhole significantly increases the coverage of the probe in reciprocal space, as shown in Fig. 2(h), which is slightly wider than the pinhole probe spectrum in Fig. 2(b). Figure 2(i) shows the corresponding reconstruction which has a clean background with no visible artifacts compared to Fig. 2(c) due to the reduced measurement time, and crisp details compared to Fig. 2(f) despite the reduced photon flux due to the enhancement of the probe spatial-frequency spectrum.

The image quality and resolution for each illuminating beam were assessed using Fourier ring correlation (FRC), a measure of Fourier correlation between two images. ${ }^{12,20,21}$ Figure 3 shows the FRC computed after subpixel alignment ${ }^{22}$ of reconstructions from independent measurements. The aforementioned artifacts in the pinhole reconstruction produce spurious self-similarities between the images and give an atypical rise of the FRC at certain high spatial frequencies.
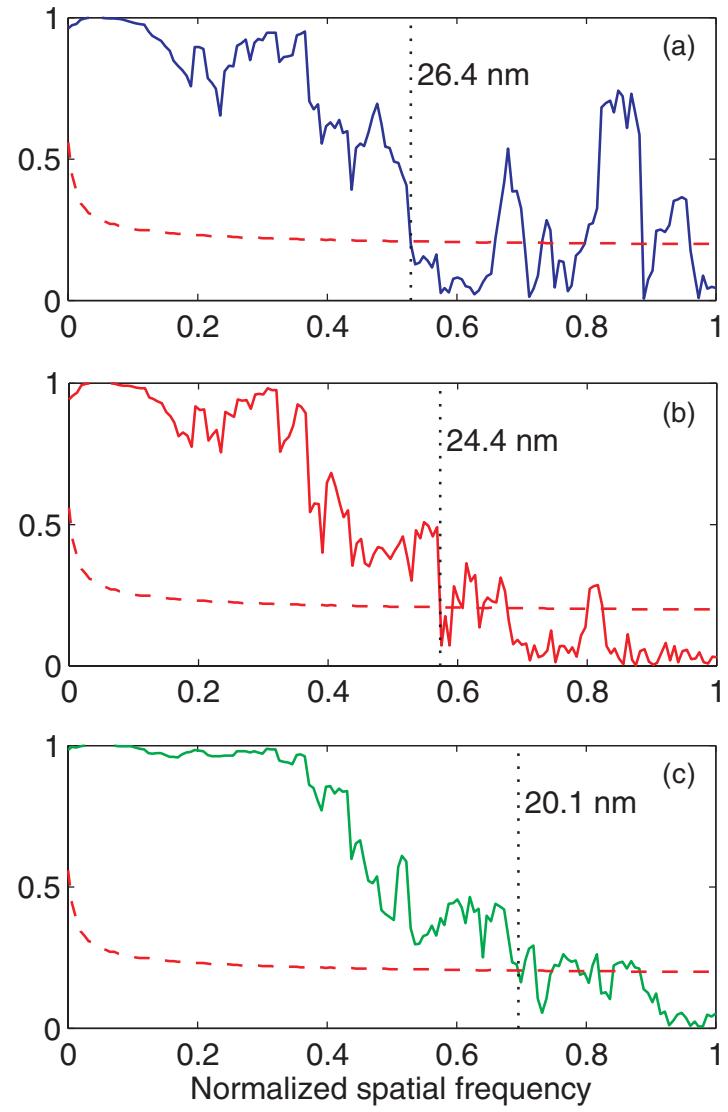

FIG. 3. (Color online) Solid curves show the FRC of reconstructions of two independent measurements for (a) pinhole illumination, (b) FZP, and (c) FZP with a pinhole clipping and perturbing the beam. Dashed curves show the $1 / 2$ bit threshold curve (Ref. 21) with the estimated half-period resolution given by the intersection with the FRC curves.

The reconstruction using the FZP with the pinhole shows an improvement in the estimated resolution and an overall increase in the FRC in Fig. 3(c), compared to the other curves. This improvement is especially noticeable around 0.2 normalized spatial frequency, which corresponds to a length scale of $70 \mathrm{~nm}$.

The approach presented here can be detrimental, however, when a resolution better than the lens numerical aperture is not expected because photons are spread out to angles where the SNR may be too low for reliable phasing. Also, as the probe spectrum is wider, the X-ray probe size increases faster along the propagation direction, meaning that the propagation distance over which the probe remains unchanged will also be reduced. It should also be noted that when introducing a perturbing element, the stability of the latter with respect to the FZP becomes important.

In conclusion we demonstrate that fidelity and resolution of ptychographic reconstructions can be enhanced by tailoring the spatial-frequency spectrum of the illuminating probe. Combining the advantages of a broad spatial-frequency spectrum and the flexibility to collect coherent flux the resolution and sensitivity can be improved with a comparable fluence, which is an important consideration for dose-sensitive samples. Furthermore, simulations suggest that this approach 
will have a larger impact for weakly scattering objects which is of special importance, for example, for imaging biological specimens at hard x-ray energies. A particularly interesting prospect is the possibility of introducing a designed perturbation to tailor the spectral properties of the probe to the contrast and desired resolution of the sample under study.
Lastly, the results presented here are not limited to $\mathrm{x}$ rays and could be useful for the application of ptychography for optical $^{23}$ and electron ${ }^{24}$ microscopy. Besides flux collection efficiency the spatial-frequency spectrum of the illumination probe, produced either by optics or enhanced by a suitable perturbation, should be a design parameter of a microscope based on ptychography. *manuel.guizar-sicairos@psi.ch

${ }^{\dagger}$ Current address: Argonne National Laboratory, Argonne, Illinois 60439, USA.

${ }^{1}$ H. M. L. Faulkner and J. M. Rodenburg, Phys. Rev. Lett. 93, 023903 (2004).

${ }^{2}$ P. Thibault, M. Dierolf, A. Menzel, O. Bunk, C. David, and F. Pfeiffer, Science 321, 379 (2008)

${ }^{3}$ M. Dierolf, A. Menzel, P. Thibault, P. Schneider, C. M. Kewish,

R. Wepf, O. Bunk, and F. Pfeiffer, Nature (London) 467, 436 (2010).

${ }^{4}$ M. Guizar-Sicairos, A. Diaz, M. Holler, M. S. Lucas, A. Menzel, R. A. Wepf, and O. Bunk, Opt. Express 19, 21345 (2011).

${ }^{5}$ M. Guizar-Sicairos and J. R. Fienup, Opt. Express 16, 7264 (2008).

${ }^{6}$ C. T. Putkunz, J. N. Clark, D. J. Vine, G. J. Williams, M. A. Pfeifer, E. Balaur, I. McNulty, K. A. Nugent, and A. G. Peele, Phys. Rev. Lett. 106, 013903 (2011).

${ }^{7}$ J. Miao, P. Charalambous, J. Kirz, and D. Sayre, Nature (London) 400, 342 (1999).

${ }^{8}$ C. M. Kewish, P. Thibault, M. Dierolf, O. Bunk, A. Menzel, J. Vila-Comamala, K. Jefimovs, and F. Pfeiffer, Ultramicroscopy 110, 325 (2010).

${ }^{9}$ A. Schropp, P. Boye, J. M. Feldkamp, R. Hoppe, J. Patommel, D. Samberg, S. Stephan, K. Giewekemeyer, R. N. Wilke, T. Salditt, J. Gulden, A. P. Mancuso, I. A. Vartanyants, E. Weckert, S. Schöder, M. Burghammer, and C. G. Schroer, Appl. Phys. Lett. 96, 091102 (2010).

${ }^{10}$ C. M. Kewish, M. Guizar-Sicairos, C. Liu, J. Qian, B. Shi, C. Benson, A. M. Khounsary, J. Vila-Comamala, O. Bunk, J. R. Fienup, A. T. Macrander, and L. Assoufid, Opt. Express 18, 23420 (2010).
${ }^{11}$ M. Guizar-Sicairos, S. Narayanan, A. Stein, M. Metzler, A. R. Sandy, J. R. Fienup, and K. Evans-Lutterodt, Appl. Phys. Lett. 98, 111108 (2011).

${ }^{12}$ J. Vila-Comamala, A. Diaz, M. Guizar-Sicairos, A. Mantion, C. M. Kewish, A. Menzel, O. Bunk, and C. David, Opt. Express 19, 21333 (2011).

${ }^{13}$ B. C. McCallum and J. M. Rodenburg, Ultramicroscopy 45, 371 (1992).

${ }^{14}$ H. N. Chapman, Ultramicroscopy 66, 153 (1996).

${ }^{15}$ J. Vila-Comamala, S. Gorelick, V. A. Guzenko, and C. David, J. Vac. Sci. Technol. B 29, 06F301 (2011).

${ }^{16}$ B. Henrich, A. Bergamaschi, C. Brönnimann, R. Dinapoli, E. F. Eikenberry, I. Johnson, M. Kobas, P. Kraft, A. Mozzanica, and B. Schmitt, Nucl. Instrum. Methods Phys. Res., Sect. A 607, 247 (2009).

${ }^{17}$ P. Thibault, M. Dierolf, O. Bunk, A. Menzel, and F. Pfeiffer, Ultramicroscopy 109, 338 (2009).

${ }^{18}$ S. Gorelick, J. Vila-Comamala, V. A. Guzenko, R. Barrett, M. Salomé, and C. David, J. Synchrotron Radiat. 18, 442 (2011).

${ }^{19}$ G. J. Williams, H. M. Quiney, B. B. Dhal, C. Q. Tran, K. A. Nugent, A. G. Peele, D. Paterson, and M. D. de Jonge, Phys. Rev. Lett. 97, 025506 (2006).

${ }^{20}$ W. O. Saxton and W. Baumeister, J. Microsc. 127, 127 (1982).

${ }^{21}$ M. van Heel and M. Schatz, J. Struct. Biol. 151, 250 (2005).

${ }^{22}$ M. Guizar-Sicairos, S. T. Thurman, and J. R. Fienup, Opt. Lett. 33, 156 (2008).

${ }^{23}$ A. M. Maiden, J. M. Rodenburg, and M. J. Humphry, Opt. Lett. 35, 2585 (2010).

${ }^{24}$ M. J. Humphry, B. Kraus, A. C. Hurst, A. M. Maiden, and J. M. Rodenburg, Nat. Commun. 3, 730 (2012). 